Research Article

\title{
Interval Intuitionistic Fuzzy Decision Model with Abnormal Information and Its Application in Talent Selection
}

\author{
Xueer Ji $\mathbb{D}$, Lei Wang $(\mathbb{D}$, and Huifeng Xue $(\mathbb{D}$ \\ China Aerospace Academy of Systems Science and Engineering, Beijing 100048, China \\ Correspondence should be addressed to Xueer Ji; jixueer@mail.nwpu.edu.cn
}

Received 16 November 2020; Revised 5 March 2021; Accepted 15 March 2021; Published 30 March 2021

Academic Editor: Yang Li

Copyright (c 2021 Xueer Ji et al. This is an open access article distributed under the Creative Commons Attribution License, which permits unrestricted use, distribution, and reproduction in any medium, provided the original work is properly cited.

\begin{abstract}
In some complex decision-making problems such as talent selection, experts often hesitate between multiple evaluation values during their decision making and can only give a range of information due to the fuzziness and imprecision of qualitative decision-making attributes. Interval intuitionistic fuzzy sets and their decision-making methods provide a useful tool to describe the fuzziness of decision attributes and decision experts' hesitation. However, the abnormal information in the expert decision information has not been considered in the previous works; that is, some interval intuitionistic fuzzy numbers exceed the defined interval range. This kind of abnormal decision information often makes it difficult to obtain accurate decision results using the decision model. To avoid the abnormal information influence on decision-making results, the hesitancy degree-based interval intuitionistic fuzzy sets are employed to propose an adaptive correction method of abnormal information, which can correct the abnormal decision information without changing the decision preference of experts. The abnormal information correction method is utilized to construct a new interval intuitionistic fuzzy entropy by combining hesitancy and fuzziness. This provides a multiattribute decision-making method, including abnormal decision information. Finally, the effectiveness and superiority of the proposed method and decision-making model are evaluated using an application case study of talent selection.
\end{abstract}

\section{Introduction}

As an essential work of modern human resource management, talent selection plays a vital role in recruitment, promotion, reserve cadre training, and performance appraisal. In the talent selection process, the assessment content is so complex, making it difficult to evaluate the assessment indicators accurately. The conventional talent selection methods, including permutation comparison, democratic election, expert evaluation, performance evaluation, and actual investigation methods, employ the qualitative investigation or simple statistical comparison and do not have a reliable scientific basis for guiding the selection work. This leads to a lack of scientific talent evaluation results as a reference for decision making in the talent selection process. New talent selection methods are mainly based on AHP (analytic hierarchy process), fuzzy decision theory, neural network, and fuzzy combination optimization method [1-4], which can significantly improve the processing ability of fuzzy talent evaluation indicators; for example, Capaldo and Zollo utilized fuzzy logic and multiattribute decision methods to study the talent evaluation problem $[5,6]$, Nussbaum et al. employed the decision support system for talent selection [7], Rothstein and Goffin studied the personality measure application in talent selection [8], and Jessop determined the minimum deviation weight required for talent selection [9]. Although the above methods provide a solution for evaluating fuzzy indicators, the decision-making process is still based on the comprehensive judgment of experts. Experts' cognitive results show hesitation between affirmative and negative in the practical applications of the talent selection due to the lack of complicated things knowledge. The impact of this hesitant information on the selection results cannot be completely described through the existing talent selection methods. As a result, there is a specific deviation between the final selection results and the experts' decision-making opinions, making it impossible to select talents accurately and efficiently. Thus, lots of researchers have started to focus on the multiattribute 
decision analysis methods. Lai and Ishizaka utilized the multicriteria decision analysis method in talent selection process [10]. Yang et al. combined multiobjective optimization with integrated decision [11]. The paper will delve into the talent selection method based on the intuitionistic fuzzy set theory with references to the aforementioned papers.

In 1986, Atanassov presented the intuitionistic fuzzy set concept [12], considering not only membership (as the traditional fuzzy sets) but also nonmembership and hesitation. The mentioned concept provides a scientific and effective method to deal with the imprecise and uncertain information of expert decision-making opinions in talent selection. Later, Atanassov and Gargov extended the intuitionistic fuzzy set and developed the interval intuitionistic fuzzy set concept [13], which can completely describe the attribute information of things. Besides, aiming at the fuzzy attribute decisionmaking problem, $\mathrm{Xu}$ presented the integration operator of interval intuitionistic fuzzy numbers, including weighted geometric integration and weighted aggregation operators [14]. Li et al. [15] employed the perspective of interval evidence combination to establish a new interval intuitionistic fuzzy set decision-making method, and Tan et al. [16] proposed an interval intuitionistic fuzzy number ranking method based on TOPSIS (Technique for Order of Preference by Similarity to an Ideal Solution). At the same time, the determination of attribute and decision-maker weight vector in the uncertain multiattribute group decision-making problem is a research challenge that can be solved through the entropy weight method. Zhao and $\mathrm{Xu}$ [17] proposed a reasonable axiomatic condition of intuitionistic fuzzy entropy and a series of intuitionistic fuzzy entropy relations by considering both fuzziness and hesitation. Continuing their work, relevant works have been performed on axiomatic conditions of interval intuitionistic fuzzy entropy and entropy calculation relation $[18,19]$. However, since the membership and nonmembership degrees of interval-valued intuitionistic fuzzy are interval values, the fuzziness description is more complicated than intuitionistic fuzzy sets. When experts provide significant information about interval-valued intuitionistic fuzzy sets, abnormal interval-valued intuitionistic fuzzy numbers that do not meet the defined conditions may appear. The traditional interval-valued intuitionistic fuzzy decisionmaking methods cannot deal with decision information matrices containing this abnormal information. At the same time, since during the entropy calculation process of some published works, the defined axiomatic conditions of interval intuitionistic fuzzy entropy cannot be fully satisfied in extreme cases, the definition of interval intuitionistic fuzzy entropy is still an open challenge.

Therefore, this paper verifies the possibility of employing the fuzzy multiattribute group decision making in talent selection. Considering the abnormal decision-making information, an interval intuitionistic fuzzy decision-making model with abnormal information is utilized to propose a talent selection method. Firstly, the expression of interval intuitionistic fuzzy sets is analyzed to present the following assumption: the hesitancy degree in expert evaluation information is stable in a short time. The stable state of interval intuitionistic fuzzy sets is adopted to develop a method for correcting abnormal information in interval intuitionistic fuzzy sets. Secondly, the relevant definitions of hesitancy and fuzziness are utilized to illustrate why the axiomatic definition conditions of inter-region intuitionistic fuzzy entropy in the existing literature cannot be satisfied in extreme cases. A more reasonable axiomatic condition of interval intuitionistic fuzzy entropy is presented, and the interval intuitionistic fuzzy entropy expression in a finite field is proposed for applying to multiattribute decision making with completely unknown weight information. Finally, the efficiency and advantages of the proposed outlier correction method and interval intuitionistic fuzzy entropy decision-making model are investigated by applying to a talent selection application case.

The remaining parts of this study are arranged as follows. Section 2 introduces the definition and operation rules of interval intuitionistic fuzzy sets. The adaptive correction method of interval intuitionistic fuzzy sets with abnormal information is presented in Section 3. Section 4 verifies why the existing axiomatic definition conditions of inter-region direct fuzzy entropy cannot be satisfied in extreme cases and revisits it in a more reasonable axiomatic condition of interval intuitionistic fuzzy entropy. Moreover, the interval intuitionistic fuzzy entropy expression in a finite field is proposed. In Section 5, the abnormal information processing method of interval intuitionistic fuzzy set and interval intuitionistic fuzzy entropy are adopted to construct an interval intuitionistic fuzzy decision model with abnormal information. In Section 6, the proposed interval intuitionistic fuzzy decision model is applied to a practical talent selection decision making. Finally, Section 7 presents the essential contents and conclusions of this study.

\section{Interval Intuitionistic Fuzzy Set and Its Algorithm}

Definition 1 (see [12]). Let $X$ be a nonempty set; then, $A=$ $\left\{\left\langle x, \mu_{A}(x), v_{A}(x)\right\rangle \mid x \in X\right\}$ is called the intuitionistic fuzzy set, where $\mu_{A}(x) \in[0,1]$ and $\nu_{A}(x) \in[0,1]$ are the membership and nonmembership degrees of the element $x$ in $X$ belonging to $A$, respectively, satisfying the following conditions:

$$
0 \leq \mu_{A}(x)+v_{A}(x) \leq 1, \quad x \in X .
$$

Moreover, the element $x$ in $X$ belongs to the hesitation $\pi_{A}(x)$ of $A$, which can be defined as

$$
\pi_{A}(x)=1-\mu_{A}(x)-v_{A}(x), \quad x \in X .
$$

The fuzzy degree $\triangle_{A}(x)$ that the element $x$ in $X$ belongs to $A$ is described as

$$
\triangle_{A}(x)=\left|\mu_{A}(x)-v_{A}(x)\right| .
$$

For convenience, $\alpha=\left(\mu_{\alpha}, \nu_{\alpha}\right)$ is called the intuitionistic fuzzy number, where $\mu_{a} \in[0,1], \nu_{a} \in[0,1]$, and $\mu_{a}+\nu_{a} \leq 1$.

If the membership degree $\mu_{A}(x)$ and nonmembership degree $v_{A}(x)$ of the element $x$ in the intuitionistic fuzzy set belonging to $A$ are represented with interval values, then the intuitionistic fuzzy set can be extended to the interval intuitionistic fuzzy set. 
Definition 2 (see [20]). Consider that the discourse domain is denoted by $X$, and the interval intuitionistic fuzzy set $\widetilde{A}$ is defined as $\widetilde{A}=\left\{\left\langle x, \widetilde{\mu}_{\widetilde{A}}(x), \widetilde{v}_{\widetilde{A}}(x)\right\rangle \mid x \in X\right\}$, where $\widetilde{\mu}_{\tilde{A}}(x)=$ $\left[\mu_{\sim}^{L}(x), \mu_{\tilde{A}}^{R}(x)\right] \subseteq[0,1]$ and $\tilde{\nu}_{A}^{A}(x)=\left[v_{\sim}^{L}(x), v_{\tilde{A}}^{R}(x)\right] \subseteq[0,1]$ are the membership and nonmembership degrees of the element $x$ in $X$ belonging to $A$, respectively, meeting the condition $\mu_{\sim}^{R}(x)+\nu_{\sim}^{R}(x) \leq 1$. The ordered interval pair $\left\langle\widetilde{\mu}_{\tilde{A}}(x), \widetilde{v}_{\tilde{A}}(\hat{A})\right\rangle$ composed of the membership interval $\tilde{\mu}_{A}^{-}(x)$ and nonmembership interval $\widetilde{\nu}_{\tilde{A}}(x)$ is called the interval intuitionistic fuzzy number.

The hesitation $\tilde{\pi}_{A}(x)$, where the element $x$ in $X$ belongs to $\tilde{A}$, can be described as

$$
\begin{aligned}
\tilde{\pi}_{A}^{\sim}(x) & =1-\widetilde{\mu}_{A}^{\sim}(x)-\widetilde{v}_{\tilde{A}}(x) \\
& =\left[1-\mu_{\tilde{A}}^{R}(x)-\nu_{\widetilde{A}}^{R}(x), 1-\mu_{\tilde{A}}^{L}(x)-\nu_{\tilde{A}}^{L}(x)\right] .
\end{aligned}
$$

The fuzzy degree $\widetilde{\triangle} \widetilde{A}(x)$ that the element $x$ in $X$ belongs to $\widetilde{A}$ can be expressed by $\widetilde{\triangle} \underset{A}{L}(x)$ and $\widetilde{\triangle} \underset{A}{R}(x)[21]$ :

$$
\widetilde{\triangle} \underset{A}{\stackrel{L}{\sim}}(x)=|\mu \stackrel{L}{\sim}(x)-v \underset{A}{\stackrel{L}{\sim}}(x)|, \widetilde{\triangle} \underset{A}{R}(x)=\left|\mu \underset{A}{R}(x)-v_{A}^{R}(x)\right| .
$$

When the fuzzy degree $\widetilde{\triangle}_{\widetilde{A}}(x)$ indicates that the element $x$ belongs to $\widetilde{A}$, it is unnecessary to utilize the interval $[\widetilde{\triangle} \underset{A}{\sim}(x), \widetilde{\triangle} \underset{A}{R}(x)]$ because $\widetilde{\triangle} \underset{A}{L}(x)>\widetilde{\triangle} \underset{A}{R}(x)$ may occur. For example, the fuzzy degree of the interval intuitionistic fuzzy number $\langle[0.3,0.4],[0.4,0.45]\rangle$ can be calculated using relation (5), $\quad \widetilde{\triangle} \underset{A}{L}(x)=|0.3-0.4|=0.1, \quad \widetilde{\triangle} \underset{A}{R}(x)=\mid 0.4-$ $0.45 \mid=0.05$, that is, $\widetilde{\triangle} \widetilde{A}(x)>\widetilde{\triangle} \widetilde{A}(x)$.

Definition 3 (see [22]). Let $\alpha=\langle[a, b],[c, d]\rangle, \alpha_{1}=\left\langle\left[a_{1}\right.\right.$, $\left.\left.b_{1}\right],\left[c_{1}, d_{1}\right]\right\rangle$, and $\alpha_{2}=\left\langle\left[a_{2}, b_{2}\right],\left[c_{2}, d_{2}\right]\right\rangle$ be the interval intuitionistic fuzzy numbers. Now, the following operations can be applied to these numbers:

(1) Complementary operation: $\bar{\alpha}=\langle[c, d],[a, b]\rangle$.

(2) Intersection operation:

$$
\begin{aligned}
\alpha_{1} \cap \alpha_{2}=\langle & {\left[\min \left(a_{1}, a_{2}\right), \min \left(b_{1}, b_{2}\right)\right], } \\
& \left.\cdot\left[\max \left(c_{1}, c_{2}\right), \max \left(d_{1}, d_{2}\right)\right]\right\rangle .
\end{aligned}
$$

(3) Union operation:

$$
\begin{aligned}
\alpha_{1} \bigcup \alpha_{2}=\langle & \left\langle\max \left(a_{1}, a_{2}\right), \max \left(b_{1}, b_{2}\right)\right], \\
\cdot & {\left.\left[\min \left(c_{1}, c_{2}\right), \min \left(d_{1}, d_{2}\right)\right]\right\rangle . }
\end{aligned}
$$

(4) Sum operation:

$$
\alpha_{1} \oplus \alpha_{2}=\left\langle\left[a_{1}+a_{2}-a_{1} a_{2}, b_{1}+b_{2}-b_{1} b_{2}\right],\left[c_{1} c_{2}, d_{1} d_{2}\right]\right\rangle .
$$

(5) Multiplication:

$\alpha_{1} \otimes \alpha_{2}=\left\langle\left[a_{1} a_{2}, b_{1} b_{2}\right],\left[c_{1}+c_{2}-c_{1} c_{2}, d_{1}+d_{2}-d_{1} d_{2}\right]\right\rangle$.

(6) Scalar multiplication:

$$
\lambda \alpha=\left\langle\left[1-(1-a)^{\lambda}, 1-(1-b)^{\lambda}\right],\left[c^{\lambda}, d^{\lambda}\right]\right\rangle, \quad \lambda>0 .
$$

(7) Exponential operation:

$$
\alpha^{\lambda}=\left\langle\left[a^{\lambda}, b^{\lambda}\right],\left[1-(1-c)^{\lambda}, 1-(1-d)^{\lambda}\right]\right\rangle, \quad \lambda>0 .
$$

The weighted arithmetic average and weighted geometric average operators for the interval intuitionistic fuzzy numbers with multiple attributes can be obtained using the above operations (see [14] for details).

Definition 4 (see [14]). Let $\alpha_{i}=\left[a_{i}, b_{i}\right],\left[c_{i}, d_{i}\right], i=1,2$, $\ldots, n$ be an interval intuitionistic fuzzy number describing $n$ attribute indices of the decision unit, the corresponding weight is denoted by $w_{1}, w_{2}, \ldots, w_{n}$, and the condition $\sum_{i=1}^{n} w_{i}=1, w_{i} \geq 0, i=1,2, \ldots, n$ is satisfied. Now, the weighted arithmetic average operator IIFWA and the weighted geometric operator IIFWG of the interval intuitionistic fuzzy number can be defined as

$$
\begin{aligned}
& \operatorname{IIFWA}\left(\alpha_{1}, \alpha_{2}, \ldots, \alpha_{n}\right)=\left\langle\left[1-\prod_{i=1}^{n}\left(1-a_{i}\right)^{w_{i}}, 1-\prod_{i=1}^{n}\left(1-b_{i}\right)^{w_{i}}\right],\left[\prod_{i=1}^{n} c_{i}^{w_{i}}, \prod_{i=1}^{n} d_{i}^{w_{i}}\right]\right\rangle, \\
& \operatorname{IIFWG}\left(\alpha_{1}, \alpha_{2}, \ldots, \alpha_{n}\right)=\left\langle\left[\prod_{i=1}^{n} a_{i}^{w_{i}}, \prod_{i=1}^{n} b_{i}^{w_{i}}\right],\left[1-\prod_{i=1}^{n}\left(1-c_{i}\right)^{w_{i}}, 1-\prod_{i=1}^{n}\left(1-d_{i}\right)^{w_{i}}\right]\right\rangle .
\end{aligned}
$$

\section{Abnormal Information Processing of Interval Intuitionistic Fuzzy Sets}

In the face of complex decision-making problems, experts should give decision-making opinions on multiple attribute information of multiple decision-making objects. Since experts prefer to express their intuitive feeling with interval intuitionistic fuzzy sets, they should provide the corresponding interval intuitionistic fuzzy sets. According to Definition 2, each interval intuitionistic fuzzy set $\widetilde{A}=\left\{\left\langle x, \widetilde{\mu} \widetilde{A}(x), \widetilde{v}_{A}(x)\right\rangle \mid x \in X\right\}$ provided by experts should meet the condition $\mu_{\sim}^{R}(x)+v_{\sim}^{R}$ $(x) \leq 1$. If the membership and nonmembership of an interval intuitionistic fuzzy set appear in expert decision information, satisfying $\mu_{\tilde{A}}^{R}(x)+v_{\tilde{A}}^{R}(x)>1$, then the interval intuitionistic fuzzy set should be ${ }^{A}$ provided again by experts for abnormal situations. However, to deal with real decision-making problems, it is often difficult for experts to directly find this abnormal information while providing interval intuitionistic fuzzy sets. 
Decision makers should give feedback on the mentioned abnormal information to corresponding experts in the information processing stage to modify the abnormal interval intuitionistic fuzzy sets, which requires a lot of time and labor costs.

By analyzing the expression of interval intuitionistic fuzzy sets of experts' opinions, hesitation describes the degree to which experts' judgment information belongs to neither membership nor nonmembership, reflecting experts' cognitive level of decision objects or attributes. Due to the relative stability of the experts' cognitive level in a short time, their hesitation degree on the judgment information of specific objects or attributes is also relatively stable. If an adaptive correction method of abnormal information can be designed based on the hesitation degree of expert decision information and the abnormal interval intuitionistic fuzzy set meeting $\mu_{\sim}^{R}(x)+\nu_{\sim}^{R}(x)>1$ is mapped to the one satisfying the condition $\mu^{A}(x)+{ }^{A} v_{\sim}^{R}(x) \leq 1$, so that the hesitation degree of the mapped one is similar to that of the expert decision information, then it can be adopted as the correction information of abnormal one for decision calculation.

The analysis of the interval intuitionistic fuzzy set definition shows that the hesitation degree description is neither membership nor nonmembership degree. The hesitation degree of intuitionistic fuzzy set in relation (2) can be obtained by subtracting the real numbers corresponding to membership and nonmembership degrees, while the hesitation degree of interval intuitionistic fuzzy set in relation (4) can be calculated by subtracting the interval numbers corresponding to membership and nonmembership degrees. If interval intuitionistic fuzzy sets degenerate into intuitionistic fuzzy sets, relation (4) is converted to relation (2). This means that the hesitation degree and the correction method of interval intuitionistic fuzzy sets are a generalization of those of intuitionistic fuzzy sets.

It is assumed that the steady value of hesitation degree of expert's intuitionistic fuzzy set for a specific decision object or attribute is $\pi_{\alpha}^{\prime}$, and the expert has abnormal intuitionistic fuzzy number $\alpha=\left(\mu_{\alpha}, \nu_{\alpha}\right)$ for the decision attribute corresponding to the decision object, where $\mu_{\alpha} \in[0,1], \nu_{\alpha} \in[0,1], \mu_{\alpha}+v_{\alpha}>1$, and $\pi_{\alpha}=1-\mu_{\alpha}-\nu_{\alpha}$ describe the hesitation degrees of abnormal intuitionistic fuzzy numbers. Consider that line segments represent the membership, nonmembership, and hesitation degrees of intuitionistic fuzzy numbers. Now, the visual representation of the correction mapping $f(\theta)$ can be described with a schematic diagram shown in Figure 1.

The correction mapping of abnormal intuitionistic fuzzy numbers presented in Figure 1 should satisfy the following conditions:

$$
\left\{\begin{array}{l}
\theta=\frac{\mu_{\alpha}+\nu_{\alpha}+\pi_{\alpha}}{\mu_{\alpha}^{\prime}+v_{\alpha}^{\prime}+\pi_{\alpha}^{\prime}} \\
\mu_{\alpha}^{\prime}+v_{\alpha}^{\prime}+\pi_{\alpha}^{\prime}=1, \\
\mu_{\alpha}^{\prime}=\frac{\mu_{\alpha}}{\theta} \\
v_{\alpha}^{\prime}=\frac{v_{\alpha}}{\theta} \\
\pi_{\alpha}^{\prime}=\frac{\pi_{\alpha}}{\theta}
\end{array}\right.
$$

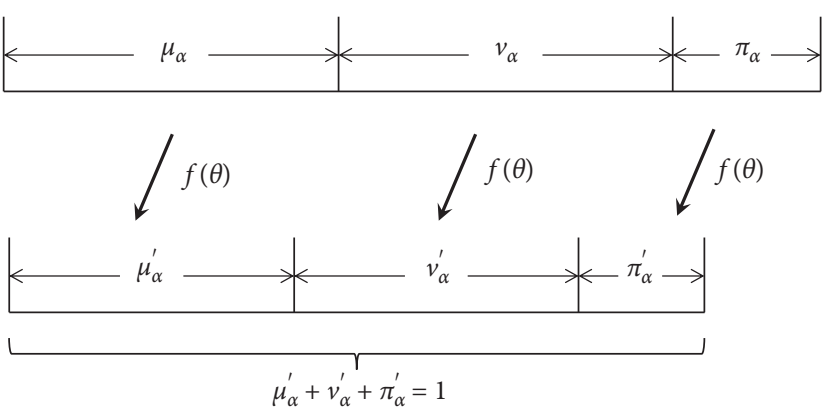

Figure 1: The schematic diagram of abnormal intuitionistic fuzzy number correction mapping.

By solving the above equations, the components of the modified intuitionistic fuzzy number $\alpha^{\prime}=\left(\mu_{\alpha}^{\prime}, \nu_{\alpha}^{\prime}\right)$ can be obtained as

$$
\begin{gathered}
\mu_{\alpha}^{\prime}=\frac{\mu_{\alpha}\left(1-\pi_{\alpha}^{\prime}\right)}{\mu_{\alpha}+\nu_{\alpha}}, \\
\nu_{\alpha}^{\prime}=\frac{\nu_{\alpha}\left(1-\pi_{\alpha}^{\prime}\right)}{\mu_{\alpha}+\nu_{\alpha}} .
\end{gathered}
$$

It can be easily verified that the modified intuitionistic fuzzy number $\alpha^{\prime}=\left(\mu_{\alpha}^{\prime}, \nu_{\alpha}^{\prime}\right)$ satisfies the condition $\mu_{\alpha}^{\prime}+\nu_{\alpha}^{\prime} \leq 1$, and the hesitation degree has a stable value $\pi_{\alpha}^{\prime}$. The mentioned method can extend the correction method of abnormal information of intuitionistic fuzzy numbers to interval ones. If the abnormal interval intuitionistic fuzzy number is denoted by $\alpha=\langle[a, b],[c, d]\rangle$, then the stable interval value of expert hesitation degree can be described as $\left[\tilde{\pi}_{\alpha}^{\prime}, \tilde{\pi}_{\alpha}^{\prime}\right]=\left[1-b^{\prime}-d^{\prime}, 1-a^{\prime}-c^{\prime}\right]$, while the correction mapping is indicated by $f\left(\theta_{L}, \theta_{R}\right)$. Now, the modified interval intuitionistic fuzzy numbers $\alpha^{\prime}=\left\langle\left[a^{\prime}, b^{\prime}\right],\left[c^{\prime}, d^{\prime}\right]\right\rangle$ can be obtained by solving the following equations:

$$
\left\{\begin{array}{l}
\theta^{L}=\frac{b+d+\tilde{\pi}_{\alpha}^{L}}{b^{\prime}+d^{\prime}+\tilde{\pi}_{\alpha}^{\prime},} \\
b^{\prime}+d^{\prime}+\tilde{\pi}_{\alpha}^{\prime}=1, \\
b^{\prime}=\frac{b}{\theta^{L}}, \\
d^{\prime}=\frac{d}{\theta^{L}}, \\
\tilde{\pi}_{\alpha}^{\prime L}=\frac{\tilde{\pi}_{\alpha}^{L}}{\theta^{L}}, \\
\theta^{R}=\frac{a+c+\tilde{\pi}_{\alpha}^{R}}{a^{\prime}+c^{\prime}+\tilde{\pi}_{\alpha}^{\prime},} \\
a^{\prime}+c^{\prime}+\tilde{\pi}_{\alpha}^{\prime}=1, \\
a^{\prime}=\frac{a}{\theta^{R}}, \\
c^{\prime}=\frac{c}{\theta^{R}}, \\
\tilde{\pi}_{\alpha}^{\prime R}=\frac{\tilde{\pi}_{\alpha}^{R}}{\theta^{R}} .
\end{array}\right.
$$


Solving relation (15) gives the components of the modified interval intuitionistic fuzzy number $\alpha^{\prime}=\left\langle\left[a^{\prime}, b^{\prime}\right],\left[c^{\prime}, d^{\prime}\right]\right\rangle$ as

$$
\begin{aligned}
& a^{\prime}=\frac{a\left(1-\tilde{\pi}_{\alpha}^{\prime R}\right)}{a+c}, \\
& b^{\prime}=\frac{b\left(1-\tilde{\pi}_{\alpha}^{\prime L}\right)}{b+d}, \\
& c^{\prime}=\frac{c\left(1-\tilde{\pi}_{\alpha}^{\prime R}\right)}{a+c}, \\
& d^{\prime}=\frac{d\left(1-\tilde{\pi}_{\alpha}^{\prime}\right)}{b+d} .
\end{aligned}
$$

Specifically, when $a=b, c=d, a^{\prime}=b^{\prime}, c^{\prime}=d^{\prime}$ are chosen in relation (16), equation (16) converts to equation (14). This means that the intuitionistic fuzzy number abnormal information correction method is a particular case of its interval one.

\section{Fuzzy Entropy of Interval Intuitionistic Fuzzy Sets}

It can be seen from Definition 3 that any interval intuitionistic fuzzy set $\widetilde{A}$ includes hesitation and fuzzy degrees as two kinds of preference expression information. The former describes neither membership nor nonmembership, while the latter describes the difference between membership and nonmembership. The fuzzy degree of the intuitionistic fuzzy set in relation (3) can be obtained through the relation of the distance between real numbers corresponding to membership and nonmembership. However, the fuzzy degree of interval intuitionistic fuzzy sets in formula (5) only calculates the left and right endpoints of membership and nonmembership interval numbers according to the distance relation between real numbers, which can neither extend the fuzzy degree of intuitionistic fuzzy sets nor reflect the fuzzy degree definition essence. Therefore, in this paper, the mentioned fuzzy degree is redefined by calculating the Euclidean distance between interval numbers.

Definition 5 (see [23]). The fuzzy degree $\widetilde{\triangle}_{\widetilde{A}}(x)$ that the element $x$ in $X$ belongs to $\widetilde{A}$ can be defined as

$$
\begin{aligned}
\widetilde{\triangle}_{\tilde{A}}(x) & =\frac{\sqrt{2}}{2} d\left(\widetilde{\mu}_{A}(x), \widetilde{v}_{A}(x)\right) \\
& =\frac{\sqrt{2}}{2} \sqrt{\left(\mu_{A}^{L}(x)-v_{\tilde{A}}^{L}(x)\right)^{2}+\left(\mu_{A}^{R}(x)-v_{A}^{R}(x)\right)^{2}},
\end{aligned}
$$

where $d\left(\widetilde{\mu}_{A}(x), \widetilde{v}_{\tilde{A}}(x)\right)$ represents the Euclidean distance between interval numbers.

For the fuzzy degree $\widetilde{\triangle} \widetilde{A}(x)$ in Definition 5 , the following natural conditions should be satisfied:
(1) $0 \leq \widetilde{\triangle}_{\widetilde{A}}(x) \leq 1$.

(2) $\widetilde{\triangle}_{\widetilde{A}}(x)=0$ if and only if $\tilde{\mu}_{\tilde{A}}(x)=\widetilde{\nu}_{\tilde{A}}(x)$.

(3) $\widetilde{\triangle}_{\widetilde{A}}(x)=1$ if and only if $\widetilde{\mu}_{A}(x)=[0,0], \widetilde{v}_{A}(x)=$ $[1,1]$, or $\widetilde{\mu}_{A}(x)=[1,1], \widetilde{v}_{A}(x)=[0,0]$.

(4) $\widetilde{\triangle}_{\widetilde{A}^{*}}(x)=\widetilde{\triangle}_{\widetilde{A}^{c}}(x)$, where $\widetilde{\triangle}_{\widetilde{A}^{c}}(x)$ is the fuzzy degree of the complement operation $\widetilde{A}^{c}$ of the interval intuitionistic fuzzy set $\widetilde{A}$.

According to Definition 5, when the interval intuitionistic fuzzy set $\widetilde{A}$ degenerates into the intuitionistic fuzzy set, the fuzzy degree relation (17) converts to the intuitionistic fuzzy set fuzzy degree relation (3), that is, the fuzzy degree $\widetilde{\triangle} \widetilde{A}(x)$ in Definition 5 is an extended version of that of intuitionistic fuzzy set in Definition 1.

Although the hesitation and fuzzy degrees in Definition 2 were employed in [17] to present the axiomatic condition of entropy of interval intuitionistic fuzzy sets, some natures are still flawed under the particular condition that the entropy value is 0 . Therefore, the mentioned axiomatic conditions are redefined using [15].

Definition 6 (see [23]). E: $\widetilde{A}(X) \longrightarrow[0,1]$ is a real numerical function of $\widetilde{\triangle} \widetilde{\sim}_{A}\left(x_{i}\right), \widetilde{\pi}_{\vec{A}}^{L}\left(x_{i}\right)$, and $\widetilde{\pi}_{A}^{R}\left(x_{i}\right)$, which satisfies the following conditions:

(1) $E(\widetilde{A})=0$ if and only if $\widetilde{A}$ is an exact set, that is, $\forall x \in X, \widetilde{\mu}_{\tilde{A}}(x)=[0,0], \widetilde{\nu}_{\widetilde{A}}(x)=[1,1]$ or $\widetilde{\mu}_{\tilde{A}}(x)=$ $[1,2], \widetilde{v}_{A}(x)=[0,0]$.

(2) $E(\widetilde{A})=1$ if and only if $\widetilde{A}=\{\langle x,[0,0],[0,0]\rangle \mid x$ $\in X\}$.

(3) $E(\widetilde{A})=E\left(\widetilde{A}^{c}\right)$, where $\widetilde{A}^{c}$ is the complement set of $\widetilde{A}$.

(4) $E(\widetilde{A})$ decreases monotonously with respect to $\widetilde{\triangle} \widetilde{A}\left(x_{i}\right)$, while it increases monotonously with respect to $\tilde{\pi}_{\tilde{A}}^{L}\left(x_{i}\right), \tilde{\pi}_{\vec{A}}^{R}\left(x_{i}\right)$.

Definition 6 can be utilized to present the entropy function theorem of interval intuitionistic fuzzy sets and reconstruct the entropy value formula for a finite field $X$.

Theorem 1 (see $[17,23])$. Let $D=\left\{\left(y, z_{1}, z_{2}\right) \mid y \subseteq[0,1]\right.$, $\left.z_{1} \subseteq[0,1], z_{2} \subseteq[0,1]\right\}$, and $F: D \longrightarrow[0,1]$ is a continuous function. Now, the entropy function $E: \widetilde{A}(X) \longrightarrow[0,1]$ can be constructed as

$$
E(\tilde{A})=\frac{1}{n} \sum_{i=1}^{n} F\left(\widetilde{\triangle} \widetilde{A}\left(x_{i}\right), \tilde{\pi}_{\tilde{A}}^{L}\left(x_{i}\right), \widetilde{\pi}_{\tilde{A}}^{R}\left(x_{i}\right)\right) .
$$

The axiomatic conditions in Definition 6 will be satisfied, if and only if the function $F$ meets the following conditions:

(1) $F\left(y, z_{1}, z_{2}\right)=0$, if and only if $y=1, z_{1}=z_{2}=0$.

(2) $F\left(y, z_{1}, z_{2}\right)=1$, if and only if $y=0, z_{1}=z_{2}=1$.

(3) The function $F$ is a monotonously decreasing function of $y$, while it is a monotonously increasing function of $z_{1}$ and $z_{2}$.

Theorem 1 was proved in [23]. 
We construct the entropy formulas in reference to $[17,24]$. According to Theorem 1, the entropy relation of the interval intuitionistic fuzzy set can be described as

$$
E(\widetilde{A})=\frac{1}{n} \sum_{i=1}^{n} \frac{\left[2-2\left(\widetilde{\triangle}_{\tilde{A}}\left(x_{i}\right)\right)^{3}+\left(\tilde{\pi}_{\vec{A}}^{L}\left(x_{i}\right)\right)^{3}+\left(\tilde{\pi}_{\vec{A}}^{R}\left(x_{i}\right)\right)^{3}\right]}{4} .
$$

\section{Multiattribute Group Decision-Making Method with Unknown Weights Using Interval Intuitionistic Fuzzy Entropy}

Let $A=\left\{A_{1}, A_{2}, \ldots, A_{m}\right\}, \quad C=\left\{C_{1}, C_{2}, \ldots, C_{n}\right\}, \quad$ and $P=\left\{P_{1}, P_{2}, \ldots, P_{k}\right\}$ be the alternative scheme, the decision attribute, and the decision expert sets, respectively. $D=\left\{D_{1}, D_{2}, \ldots, D_{k}\right\}$ describes the decision information matrix set of the expert group, where $D_{l}(l=1,2, \ldots, k)$ denotes the decision information matrix of the scheme set $A$ on the attribute set $C$ for the expert $P_{l}(l=1,2, \ldots, k)$.

$$
D_{l}=\left(\begin{array}{ccc}
d_{11}^{l} & \cdots & d_{1 n}^{l} \\
\vdots & \ddots & \vdots \\
d_{m 1}^{l} & \cdots & d_{m n}^{l}
\end{array}\right)
$$

where $\quad d_{i j}^{l}=\left\langle\left[\mu_{i j}^{l L}, \mu_{i j}^{l R}\right],\left[\nu_{i j}^{l L}, v_{i j}^{l R}\right]\right\rangle(i=1,2, \ldots, m ; j=1,2$, $\ldots, n)$ represents the interval intuitionistic fuzzy set decision information given by the expert $P_{l}$ for attribute $C_{j}$ of the scheme $A_{i}$. In the decision attribute set, if the decision attribute is a benefit, the scheme is better for higher scheme attribute values; if the decision attribute is cost type, the complementary operation of interval intuitionistic fuzzy set can be utilized to transform it into a benefit attribute value. Among them, expert and decision attribute weights are entirely unknown. Specific decision-making steps are summarized as follows:

Step 1: correction of abnormal information of the decision matrix.

(1) Screening abnormal information: traverse $k$ expert decision matrices $D_{l}(l=1,2, \ldots, k)$ and indicate whether there is abnormal interval intuitionistic fuzzy set $d_{i j}^{l}=\left\langle\left[\mu_{i j}^{l L}, \mu_{i j}^{l R}\right],\left[\nu_{i j}^{l L}, v_{i j}^{l R}\right]\right\rangle$ in the decision matrix $D_{l}$ so that $\mu_{i j}^{l R}+v_{i j}^{l R}>1$.

(2) Correction of abnormal information: suppose that there is an abnormal interval intuitionistic fuzzy set $d_{i^{\prime} j^{\prime}}^{l}$. Now, the arithmetic mean of the hesitation interval of the interval intuitionistic fuzzy set can be calculated after removing abnormal information in the $i^{\prime}-$ th row and the $j^{\prime}-$ th column of the decision matrix $D_{l}$. Let the mean value of the row hesitation interval be $\left[\pi_{i^{\prime}}^{l L}, \pi_{i^{\prime}}^{l R}\right]$, the mean value of the column hesitation interval be $\left[\pi_{j^{\prime}}^{l L}, \pi_{j^{\prime}}^{l R}\right.$, and the preference coefficient between decision object hesitation and decision attribute hesitation of the decision maker be $\gamma$ ( $\gamma$ is larger than 0.5 if decision maker hopes to keep experts' stable judgement of decision objects; $\gamma$ is less than 0.5 if decision maker hopes to keep experts' stable judgement of decision attributes; otherwise, $\gamma$ is equal to 0.5 ); then, the stable value of the hesitation interval in the abnormal information correction process is $\left[\pi_{i^{\prime} j^{\prime}}^{l L}\right.$, $\left.\pi_{i^{\prime} j^{\prime}}^{l R}\right]=\left[\gamma \pi_{i^{\prime}}^{l L}+(1-\gamma) \pi_{j^{\prime}}^{l L}, \gamma \pi_{i^{\prime}}^{l R}+(1-\gamma) \pi_{j^{\prime}}^{l R}\right]$, while the abnormal information can be corrected using relation (16).

Step 2: conversion of the cost attribute value.

For the $k$ expert decision matrices $D_{l}(l=1,2, \ldots, k)$ modified by the abnormal information, aiming at the cost attribute, the interval intuitionistic fuzzy set complement operation under this attribute can be transformed into the benefit attribute value, and the transformed $k$ expert decision matrices are indicated by $\widetilde{D}_{l}(l=1,2, \ldots, k)$.

Step 3: information integration of decision-making experts.

(1) Combine the $j$ th attribute values of the $i$ th scheme given by $k$ experts with the decision matrix $\widetilde{D}_{l}(l=$ $1,2, \ldots, k)$ to form the vector $K D_{i j}=\left(d_{i j}^{1}, d_{i j}^{1}, \ldots\right.$, $\left.d_{i j}^{l}, \ldots, d_{i j}^{k}\right)$ and calculate the corresponding entropy vector using relation (19).

(2) Calculate the weight $w_{i j}^{l}$ for each expert on the $j$ th attribute of the $i$ th scheme.

(3) The interval intuitionistic fuzzy set weighted aggregation operator [19] is employed to integrate $k$ expert decision matrices $D_{l}(l=1,2, \ldots, k)$ and obtain the expert comprehensive decision matrix A D.

(4) $A D=\left(\begin{array}{ccc}d_{11} & \cdots & d_{1 n} \\ \vdots & \ddots & \vdots \\ d_{m 1} & \cdots & d_{m n}\end{array}\right), d_{i j}=\sum_{l=1}^{k} w_{i j}^{l} d_{i j}^{l}, i=1,2, \ldots$, $m ; j=1,2, \ldots, n$.

Step 4: integration of decision attribute information.

(1) Employ relation (19) to calculate the entropy matrix $E(A D)$ of the comprehensive decision matrix $A D$ as

$$
E(A D)=\left(\begin{array}{ccc}
E\left(d_{11}\right) & \cdots & E\left(d_{1 n}\right) \\
\vdots & \ddots & \vdots \\
E\left(d_{m 1}\right) & \cdots & E\left(d_{m n}\right)
\end{array}\right)
$$

(2) Calculate the attribute weight vector $w=\left(w_{1}, w_{2}\right.$, $\left.\ldots, w_{n}\right): \quad w_{j}=\left(1-\sum_{i=1}^{m} E\left(d_{i j}\right)\right) /\left(n-\sum_{j^{\prime}=1}^{n} \sum_{i=1}^{m}\right.$ $\left.E\left(d_{i j^{\prime}}\right)\right), j=1,2, \ldots, n$.

(3) The weighted aggregation operator of interval intuitionistic fuzzy sets is utilized to weight and aggregate the comprehensive decision matrix $A D$ to obtain the integration result as $\mathrm{SAD}=\left(\mathrm{SAD}_{1}, \mathrm{SAD}_{2}, \ldots, \mathrm{SAD}_{i}, \ldots, \mathrm{SAD}_{m}\right)$, where $\mathrm{SAD}_{i}=\sum_{j=1}^{k} d_{i j} w_{j}, i=1,2, \ldots, m$. 
Step 5: ranking the advantages and disadvantages of decision objects.

(1) Relation (19) can be employed to calculate the entropy vector $E(\mathrm{SAD})$ of the integration result $\mathrm{SAD}$ as

$$
E(\mathrm{SAD})=\left(E\left(\mathrm{SAD}_{1}\right), E\left(\mathrm{SAD}_{2}\right), \ldots, E\left(\mathrm{SAD}_{m}\right)\right)
$$

(2) According to the interval intuitionistic fuzzy entropy definition, the lower values for the scheme's entropy in the integration result reflect a superior scheme.

\section{Application of Interval Intuitionistic Fuzzy Entropy-Based Multiattribute Decision- Making Model in Talent Selection}

To solve the recruitment problem of company A, for example, three experts in company A evaluated 3 candidates and picked the top one among them with 4 evaluation indices: thinking logic,innovation capacity, management coordination, and decision-making ability. In the decision process, $A=\left\{A_{1}, A_{2}, A_{3}\right\}, C=\left\{C_{1}, C_{2}, C_{3}, C_{4}\right\}, P=\left\{P_{1}, P_{2}, P_{3}\right\}$, and $D=\left\{D_{1}, D_{2}, D_{3}\right\}$ describe the scheme, the decision attribute, the decision expert, and the expert decision matrix sets, respectively. The experts employed interval intuitionistic fuzzy sets to express the interviewers' comprehensive evaluation to obtain the interval intuitionistic fuzzy set decision matrix $D_{l}(l=1,2,3)$ of expert $P_{l}(l=1,2,3)$ on scheme set $A$ on attribute set $C$, while the matrix element $d_{i j}^{l}=\left\langle\left[\mu_{i j}^{l L}\right.\right.$, $\left.\left.\mu_{i j}^{l R}\right],\left[\nu_{i j}^{l L}, v_{i j}^{l R}\right]\right\rangle(i=1,2,3 ; j=1,2,3,4)$ represents the satisfaction and dissatisfaction of expert $P_{l}$ on scheme $A_{i}$ on attribute $C_{j}$. The specific data of the decision matrix are given as follows:

$$
\begin{aligned}
& D_{1}=\left(\begin{array}{ccccc}
\langle[0.70,0.80],[0.05,0.15]\rangle & \langle[0.75,0.80],[0.05,0.10]\rangle\langle[0.35,0.45],[0.40,0.50]\rangle\langle[0.60,0.70],[0.15,0.20]\rangle \\
\langle[0.55,0.65],[0.20,0.30]\rangle\langle[0.68,0.76],[0.15,0.21]\rangle\langle[0.70,0.80],[0.05,0.10]\rangle\langle[0.45,0.50],[0.05,0.15]\rangle \\
\langle[0.75,0.80],[0.10,0.15]\rangle\langle[0.42,0.48],[0.45,0.55]\rangle\langle[0.50,0.65],[0.25,0.30]\rangle\langle[0.70,0.80],[0.05,0.10]\rangle
\end{array}\right), \\
& D_{2}=\left(\begin{array}{llll}
\langle[0.70,0.76],[0.10,0.15]\rangle & \langle[0.82,0.85],[0.05,0.10]\rangle & \langle[0.30,0.35],[0.40,0.50]\rangle & \langle[0.40,0.45],[0.35,0.40]\rangle \\
\langle[0.55,0.60],[0.25,0.30]\rangle\langle[0.65,0.70],[0.20,0.25]\rangle & \langle[0.78,0.85],[0.05,0.10]\rangle & \langle[0.62,0.75],[0.10,0.15]\rangle \\
\langle[0.70,0.85],[0.05,0.10]\rangle & \langle[0.60,0.65],[0.25,0.30]\rangle & \langle[0.65,0.70],[0.15,0.20]\rangle\langle[0.70,0.74],[0.15,0.20]\rangle
\end{array}\right), \\
& D_{3}=\left(\begin{array}{llll}
\langle[0.86,0.92],[0.05,0.10]\rangle & \langle[0.70,0.75],[0.05,0.15]\rangle\langle[0.45,0.50],[0.30,0.35]\rangle\langle[0.60,0.65],[0.25,0.35]\rangle \\
\langle[0.70,0.75],[0.05,0.10]\rangle\langle[0.65,0.76],[0.10,0.15]\rangle\langle[0.65,0.73],[0.12,0.20]\rangle\langle[0.55,0.63],[0.05,0.15]\rangle \\
\langle[0.55,0.60],[0.25,0.30]\rangle\langle[0.50,0.60],[0.25,0.35]\rangle\langle[0.65,0.75],[0.15,0.22]\rangle\langle[0.50,0.55],[0.15,0.20]\rangle
\end{array}\right) .
\end{aligned}
$$

6.1. Talent Selection Analysis of Multiattribute DecisionMaking Model Using Abnormal Information Modification and Interval Intuitionistic Fuzzy Entropy

Step 1. After inspection, it can be concluded that $D_{1}(3,2)$ and $D_{3}(1,1)$ are abnormal interval intuitionistic fuzzy sets in the expert decision information matrix, and the preference coefficient proposed by the expert is $\gamma=0.5$. According to the correction method of abnormal information in Section 5, the corrected interval intuitionistic fuzzy numbers of $D_{1}(3,2)$ and $D_{3}(1,1)$ are obtained as $\langle[0.39,0.44]$, [0.41, $0.50]\rangle$ and $\langle[0.74,0.81],[0.04,0.09]\rangle$, respectively. Moreover, the corrected decision information matrix is still represented as $D=\left\{D_{1}, D_{2}, D_{3}\right\}$.

Step 2. The four comprehensive evaluation attributes are all benefit ones without any need to transform.

Step 3. The entropy relation (19) is employed to calculate each expert's weight on the $j$ th attribute of the $i$ th scheme, while the expert decision matrix is integrated to obtain the expert comprehensive decision matrix $A D$ as

$$
\begin{aligned}
& \text { A } D=\left(\begin{array}{cc}
\langle[0.7133,0.7911],[0.0590,0.1243]\rangle & \langle[0.7649,0.8067],[0.0500,0.1133]\rangle \\
\langle[0.6144,0.6792],[0.1264,0.1974]\rangle & \langle[0.6604,0.7424],[0.1428,0.1976]\rangle \\
\langle[0.6845,0.7814],[0.1003,0.1570]\rangle & \langle[0.5045,0.5722],[0.2947,0.3728]\rangle
\end{array}\right. \\
& \langle[0.3698,0.4367],[0.3780,0.4439]\rangle\langle[0.5451,0.6174],[0.2324,0.2999]\rangle \\
& \langle[0.7182,0.8021],[0.0832,0.1423]\rangle\langle[0.5504,0.6485],[0.0643,0.1500]\rangle \\
& \langle[0.6088,0.7045],[0.1759,0.2346]\rangle\langle[0.6518,0.7234],[0.0994,0.1543]\rangle)
\end{aligned}
$$


TABLE 1: Calculation results of three decision models.

\begin{tabular}{lcc}
\hline Decision model & Interviewer ranking results & Winner \\
\hline Outlier correction decision model (the proposed method in this article) & $A_{2}>A_{3}>A_{1}$ & $A_{2}$ \\
Decision model 1 & $A_{2}>A_{1}>A_{3}$ & $A_{2}>A_{3}>A_{1}$ \\
Decision model 2 & $A_{2}>A_{2}$ \\
\hline
\end{tabular}

Step 4. By using the comprehensive decision matrix $A D$, the attribute weights can be calculated as

$$
w=\left(w_{1}, w_{2}, w_{3}, w_{4}\right)=(0.1747,0.2213,0.2875,0.3165) .
$$

The SAD results after integrating are given by

$$
\begin{aligned}
& \mathrm{SAD}_{1}=\langle[0.6018,0.6692],[0.1497,0.2320]\rangle, \\
& \mathrm{SAD}_{2}=\langle[0.6403,0.7262],[0.0930,0.1648]\rangle, \\
& \mathrm{SAD}_{3}=\langle[0.6174,0.7020],[0.1492,0.2122]\rangle .
\end{aligned}
$$

Step 5. According to the aggregation result $\mathrm{SAD}=\left(\mathrm{SAD}_{1}\right.$, $\mathrm{SAD}_{2}, \mathrm{SAD}_{3}$ ), the corresponding entropy values are calculated as follows:

$$
\begin{aligned}
& E\left(\mathrm{SAD}_{1}\right)=0.4601 \\
& E\left(\mathrm{SAD}_{2}\right)=0.4198 \\
& E\left(\mathrm{SAD}_{3}\right)=0.4483
\end{aligned}
$$

As previously discussed, the smaller the entropy value is, the better the result is. Accordingly, the ability ranks of the three interviewers are given as follows: $A_{2}>A_{3}>A_{1}$.

The proposed interval intuitionistic fuzzy set abnormal information correction method and decision model can effectively solve complex decision-making problems in real applications like abnormal expert decision-making information.

6.2. Comparative Analysis of Decision-Making Methods. To further evaluate the advantages of the proposed multiattribute decision-making method, two new decisionmaking models are constructed in this section to deal with the aforementioned talent selection problems and compare the results of these three decision-making models. After eliminating the abnormal information processing steps form the proposed decision model, decision model 1 is constructed. Decision model 2 can be obtained by replacing the entropy formula of inter-region intuitionistic fuzzy sets in the proposed decision model with the decision model after entropy formula (28) in reference [24].

$$
E_{1}(\tilde{A})=\frac{1}{n} \sum_{i=1}^{n} \frac{\left(1-\widetilde{\triangle}_{\widetilde{A}}\left(x_{i}\right)\right)\left(2+\tilde{\pi}_{\tilde{A}}^{L}\left(x_{i}\right)+\tilde{\pi}_{\tilde{A}}^{R}\left(x_{i}\right)\right)}{4} .
$$

The aforementioned talent selection case employs decision-making model 1 to calculate the following results:

$$
\begin{aligned}
w & =\left(w_{1}, w_{2}, w_{3}, w_{4}\right) \\
& =(0.1421,0.2397,0.3113,0.3069) \\
E\left(\mathrm{SAD}_{1}\right) & =0.4487 \\
E\left(\mathrm{SAD}_{2}\right) & =0.4187 \\
E\left(\mathrm{SAD}_{3}\right) & =0.450
\end{aligned}
$$

It can be concluded from the calculation results that although the final decision results can be obtained by direct use of the decision information data containing the intuitionistic fuzzy number of the abnormal interval, there is a significant difference between the calculated attribute weight vector and one obtained after correcting the abnormal information. Finally, the ranking result of the interviewers $A_{1}$ and $A_{3}$ obtained from model 1 is that $A_{1}>A_{3}$. This completely contradicts the proposed method results.

The aforementioned talent selection case adopts decision-making model 2 to calculate the following results:

$$
\begin{aligned}
w & =\left(w_{1}, w_{2}, w_{3}, w_{4}\right) \\
& =(0.1394,0.2397,0.2840,0.3369) ; \\
E\left(\mathrm{SAD}_{1}\right) & =0.2687 \\
E\left(\mathrm{SAD}_{2}\right) & =0.2278 \\
E\left(\mathrm{SAD}_{3}\right) & =0.2659
\end{aligned}
$$

According to the decision result of model 2, although the decision attribute vector and the entropy value of the selected person's comprehensive ability are different from the corresponding ones obtained from this method, the primary trend of the data result remains the same.

Decision results are summarized in Table 1 to visually analyze the differences in the calculation results of the three decision models.

According to the calculation results of the three decision-making models (see Table 1), although the final candidate for all decision models is $A_{1}$, the interviewees' ranking results are different. Among them, the interviewer ranking result calculated by decision model 1 is completely different from the other two ones. Due to the lack of abnormal data processing in the decision information, the result of the interviewers $A_{2}$ and $A_{3}$ is the opposite of the proposed method. Simultaneously, although the calculation result of decision model 2 is similar to the proposed method, the proposed axiomatic definition and calculation formula of interval intuitionistic fuzzy entropy can meet all extreme conditions and provide superior applicability. Therefore, the proposed multiattribute decision-making model combining interval intuitionistic fuzzy number 
anomaly information correction method with entropy theory can effectively solve the multiattribute decisionmaking problem with unknown weights containing anomalous information and has various applications. Through the comparative analysis of three decision-making models, the method proposed in this paper avoids the manpower, time, and economic costs caused by human factors. Compared with the traditional decision-making model, it has the advantages of efficient algorithm, strong realizability, and wide application fields.

\section{Conclusion}

This paper focuses on the situation that abnormal interval intuitionistic fuzzy sets may appear in expert decisionmaking information in the process of multiattribute expert group decision making in talent selection. The main innovations of the paper are listed below:

(1) The hesitation degree is utilized to design a correction method of abnormal interval intuitionistic fuzzy set, which is capable of mapping abnormal interval intuitionistic fuzzy set to standard information based on the constant preference of experts in decision making. The correction method of abnormal interval intuitionistic fuzzy set is utilized to solve the talent selection problem based on the multiattribute decision making. This method can make up for the lack of ability for current multiattribute methods to deal with abnormal information. This method can avoid possible changes from abnormal information. The proposed method in the paper can correct abnormal information. The correction process mainly refers to the experts' judgement information. The method cannot accurately know experts' long term judgement preferences. In consecutive research, this problem needs to be further improved.

(2) This paper calculates the distance of interval numbers and the ambiguity of interval intuitionistic fuzzy sets. Then, the hesitation degree is combined with the fuzzy degree of interval intuitionistic fuzzy sets to modify the previous definition of interval intuitionistic fuzzy entropy and construct its relation in a finite field when limit condition cannot be satisfied. The correction method of abnormal interval intuitionistic fuzzy set and interval intuitionistic fuzzy entropy are utilized to solve the talent selection problem based on the multiattribute decision making. The entropy weight method is adopted to propose a multiattribute group decision-making method with a completely unknown weight, where its feasibility and effectiveness are verified through a numerical example.

(3) The proposed method can avoid the result deviation and some invalid data caused by the intuitionistic fuzzy number in the abnormal interval during the systematic evaluation of experts in a talent selection procedure. Compared with the traditional decision- making method, the adaptive abnormal data correction method greatly improves the efficiency of talent selection and makes the process of acquiring selection data and results more accurate and fast. This considerably improves the talent selection and provides more accurate data selection and results in talent selection methods. In the future, the multiattribute group decision model method based on interval intuitionistic fuzzy entropy can also be extended to many practical problems such as performance evaluation, professional assessment, and so on.

\section{Data Availability}

The data used to support the findings of this study are included within the article.

\section{Disclosure}

The funders had no role in the design of the study; in the collection, analyses, or interpretation of data; in the writing of the manuscript; or in the decision to publish the results.

\section{Conflicts of Interest}

The authors declare that they have no conflicts of interest.

\section{Authors' Contributions}

HX was responsible for supervision, project administration, and funding acquisition. XJ and LW contributed to formal analysis and methodology. XJ was responsible for software, validation, investigation, resources, data curation, original draft preparation, review and editing, and visualization. XJ and HX were responsible for conceptualization. All authors have read and agreed to the published version of the manuscript.

\section{Acknowledgments}

This research was funded by the "Hsue-Shen Tsien's Life and Thought Research" Organization of Shanghai Jiao Tong University in 2019 and National Natural Science Foundation of China (U1501253).

\section{References}

[1] X. Yang and P. Zhang, "Comprehensive evaluation of the quality of administrative leaders," Journal of Shaanxi University of Science and Technology, vol. 4, pp. 128-133, 2004.

[2] X. Du and Q. Bai, "Decision support system for talent selection based on fuzzy comprehensive evaluation model," Journal of Chongqing University (Nature), vol. 20, no. 1, pp. 112-116, 1997.

[3] X. Yang and P. Zhang, "Group decision support system for senior administrative personnel selection scheme based on neural network," Statistical Research, vol. 7, pp. 66-68, 2005.

[4] C. Zhang, "Problems and countermeasures of the open selection system of leading cadres of the party and government," Theoretical Discussion, vol. 1, pp. 159-162, 2007. 
[5] G. Capaldo and G. Zollo, "Applying fuzzy logic to personnel assessment: a case study," Omega, vol. 29, no. 6, pp. 585-597, 2001.

[6] F. Aleskerov, H. Ersel, and R. Yolalan, "Personnel allocation among bank branches using a two-stage multi-criterial approach," European Journal of Operational Research, vol. 148, no. 1, pp. 116-125, 2003.

[7] M. Nussbaum, M. Singer, R. Rosas et al., "Decision support system for conflict diagnosis in personnel selection," Information \& Management, vol. 36, no. 1, pp. 55-62, 1999.

[8] M. G. Rothstein and R. D. Goffin, "The use of personality measures in personnel selection: what does current research support?" Human Resource Management Review, vol. 16, no. 2, pp. 155-180, 2006.

[9] A. Jessop, "Minimally biased weight determination in personnel selection," European Journal of Operational Research, vol. 153, no. 2, pp. 433-444, 2004.

[10] Y.-L. Lai and A. Ishizaka, "The application of multi-criteria decision analysis methods into talent identification process: a social psychological perspective," Journal of Business Research, vol. 109, 2020.

[11] L. Yang, J. Wang, D. Zhao, G. Li, and C. Chen, "A two-stage approach for combined heat and power economic emission dispatch: combining multi-objective optimization with integrated decision making," Energy, vol. 162, 2018.

[12] K. Atannassov, "Intuitionistic fuzzy set," Fuzzy Sets and Systems, vol. 20, pp. 87-96, 1986.

[13] K. Atannassov and G. Gargov, "Interval-valued intuitionistic fuzzy sets," Fuzzy Sets and Systems, vol. 31, pp. 343-349, 1989.

[14] Z. Xu, "Integrated method of interval intuitionistic fuzzy information and its application in decision," Control And Decision, vol. 22, pp. 215-219, 2007.

[15] Y. Li, X. Deng, and Y. Deng, "A new interval intuitionistic fuzzy set decision-making method: the perspective of interval evidence combination," Control and Decision-Making, vol. 29, pp. 1143-1147, 2014.

[16] J. Tan, C. Zhu, X. Zhang, and L. Zhu, "Interval intuitionistic fuzzy number ranking method based on topsis," Control and Decision, vol. 30, pp. 2014-2018, 2015.

[17] N. Zhao and Z. S. Xu, "Entropy measures for interval-valued intuitionistic fuzzy information from a comparative perspective and their application to decision making," Informatica, vol. 27, pp. 203-229, 2015.

[18] Y. J. Zhang, P. J. Ma, X. H. Su, and C. P. Zhang, "Entropy on interval-valued intuitionistic fuzzy sets and its application in multi-attribute decision making," International Conference on Information Fusion, vol. 292, pp. 1-25, 2011.

[19] Q. Zhang, H. Xing, F. Liu, J. Ye, and P. Tang, "Some new entropy measures for interval-valued intuitionistic fuzzy sets based on distances and their relationships with similarity and inclusion measures," Information Sciences, vol. 283, pp. 55-69, 2014.

[20] K. T. Atanassov, "Operators over interval valued intuitionistic fuzzy sets," Fuzzy Sets and Systems, vol. 64, no. 2, pp. 159-174, 1994.

[21] K. Atanassov and G. Gargov, "Interval valued intuitionistic fuzzy sets," Fuzzy Sets and Systems, vol. 31, no. 3, pp. 343-349, 1989.

[22] R. Gong and L. Ma, "Score function and exact function based on interval intuitionistic fuzzy number and their applications," System Engineering Theory and Practice, vol. 39, pp. $463-475,2019$.

[23] S. Yang, Z. Tian, and Y. Lyu, "Fuzzy entropy based on interval-valued intuitionistic fuzzy set and its application,"
Journal of Guangxi University (Natural Science Edition), vol. 43, pp. 2478-2489, 2018.

[24] K. Qu, L. Tang, and W. Rong, "Construction of interval intuitionistic fuzzy set entropy and its basic properties," Journal of Chongqing University of Arts and Sciences (Natural Science Edition), vol. 29, pp. 21-24, 2010. 